Mens

Revue d'histoire intellectuelle et culturelle

mens

\title{
Silences entrecoupés, bourdonnements et papillonnage : au-delà du mutisme apparent de l'histoire littéraire des femmes (1840-1860)
}

\section{Julie Roy}

Volume 19, numéro 1-2, automne 2018, printemps 2019

Les années 1840 : rupture ou réarticulation des possibles?

URI : https://id.erudit.org/iderudit/1070074ar

DOI : https://doi.org/10.7202/1070074ar

Aller au sommaire du numéro

Éditeur(s)

Centre de recherche en civilisation canadienne-française

ISSN

1492-8647 (imprimé)

1927-9299 (numérique)

Découvrir la revue

Citer cet article

Roy, J. (2018). Silences entrecoupés, bourdonnements et papillonnage : au-delà du mutisme apparent de l'histoire littéraire des femmes (1840-1860). Mens, 19(1-2), 185-208. https://doi.org/10.7202/1070074ar
Résumé de l'article

La liste des femmes qui ont participé à la vie littéraire au xIx ${ }^{\mathrm{e}}$ siècle s'est considérablement allongée au cours des dernières années. En dépit de la relecture des textes littéraires les plus connus selon de nouvelles perspectives et la découverte de nouveaux corpus, les années 1840 et 1850 demeurent encore peu fréquentées par les chercheurs. Cet article offre une exploration de ces deux décennies par le relais d'un échantillonnage de textes parus dans la presse périodique, dont La Ruche littéraire, et par l'observation de l'apport des premiers journaux de couventines à la création d'un habitus littéraire chez les filles. 


\section{Silences entrecoupés, bourdonnements et papillonnage: au-delà du mutisme apparent de l'histoire littéraire des femmes (1840-1860)}

Julie Roy

Bibliothèque et Archives Canada

\section{Résumé}

La liste des femmes qui ont participé à la vie littéraire au XIX ${ }^{e}$ siècle s'est considérablement allongée au cours des dernières années. En dépit de la relecture des textes littéraires les plus connus selon de nouvelles perspectives et la découverte de nouveaux corpus, les années 1840 et 1850 demeurent encore peu fréquentées par les chercheurs. Cet article offre une exploration de ces deux décennies par le relais d'un échantillonnage de textes parus dans la presse périodique, dont $\mathrm{La}$ Ruche littéraire, et par l'observation de l'apport des premiers journaux de couventines à la création d'un habitus littéraire chez les filles.

\section{Abstract}

The list of women who participated in literary life during the 19th century has grown significantly in recent years. Despite the new perspectives through which we read the best-known texts and the discovery of new corpus, the 1840s and 1850s have received little consideration from researchers. This article offers an exploration of these two decades by presenting a sampling of texts published in the periodical press during that period, including La Ruche litterraire, and by observing the contribution of the first "journaux de couventines" to the creation of a literary habitus for the girls. 
Des recherches récentes réalisées sur diverses périodes du XIX ${ }^{\mathrm{e}}$ siècle ont permis de revoir peu à peu la liste des femmes qui ont participé à la vie littéraire et se sont fait apprécier de leurs contemporains. La publication du roman Angéline de Montbrun, de Laure Conan, au début des années 1880, même s'il continue d'être un marqueur important de l'histoire littéraire des femmes, n'est plus l'unique jalon permettant de définir un avant et un après. Dans les années 1810 et 1820, plusieurs lettres à l'éditeur et poésies anonymes et pseudonymes ont été publiées dans la Gazette des Trois-Rivières et le Spectateur canadien, notamment ${ }^{1}$. Odile Cherrier alias Anaïs, la première femme dont on connaît la véritable identité, a fait paraitre des traductions et des poésies dans Le Populaire à la fin des années 1830 et figure dans Le répertoire national de James Huston publié en $1848^{2}$. Un roman comme Les fiancés d'outre-tombe de Clara Chagnon, paru en feuilleton dans la Revue canadienne en 1869 et recensé par Edmond Lareau dans son Histoire de la littérature canadienne ${ }^{3}$, ou encore les nombreuses poésies d'Élise B. Larivière, publiées dans divers journaux à partir de la fin des années $1860^{4}$, permettent de multiplier les voix, de nuancer le discours et de mieux comprendre les enjeux qui ont modelé l'apport des femmes à la vie littéraire au cours de ce

1 Pour une analyse du débat entre Adélaïde et l'Hermite, voir Julie Roy, «La lettre au journal entre réalité et fiction: Adélaïde contre l'Hermite dans la Gazette des Trois-Rivières en 1819", dans Nova Doyon et Julie Roy (dir.), Le littéraire à l'ouvre dans les périodiques québécois du XIX siècle, Centre de recherche interuniversitaire sur la littérature et la culture québécoises (CRILCQ), Université de Montréal, 2005, p. 37-54, coll. «Nouveaux cahiers de recherche».

2 Huston reproduit «Une scène à St. Domingue», une traduction d'un récit paru dans un journal américain.

3 Voir Julie Roy, "Laure Conan et les "Fiancés d'outre-tombe” de Mlle Chagnon: une filiation littéraire inédite», dans Hélène Jacques, Karim Larose et Sylvano Santini (dir.), Sens communs: expérience et transmission dans la littérature québécoise, Montréal, Éditions Nota bene, 2007, p. 259-282, coll. «Convergences».

4 Voir Julie Roy, «De Catherine-Françoise de Céloron à Élise B. Larivière: la sociabilité comme creuset et faire-valoir de l'activité épistolaire et littéraire des femmes au XIX ${ }^{\mathrm{e}}$ siècle», dans Marie-Andrée Beaudet et Mylène Bédard (dir.), Relire le XIX siècle québécois à travers ses discours épistolaires, Montréal, Éditions Nota bene, 2016, p. 39-61, coll. «Romantismes». 
siècle. C'est sans compter la mise au jour d'œuvres littéraires restées manuscrites et de correspondance qui ouvrent les perspectives en donnant sa juste place à l'écriture dite privée dans un contexte où la publication, au sens moderne du terme, n'est pas l'unique moyen de reconnaissance pour les femmes qui écrivent, comme en témoignent les poésies d'une Louise-Amélie Panet Berczy, redécouvertes dans des albums, et ses journaux personnels 5 .

Fruits de recherches souvent laborieuses et de dépouillements minutieux dans les fonds d'archives et dans la presse, les corpus qui permettent de reconstituer la vie littéraire des femmes au XIX ${ }^{\mathrm{e}}$ siècle demeurent difficiles à établir. Dans ce siècle encore malgré tout peu fréquenté, les décennies 1840 et 1850 constituent toujours d'ailleurs une sorte de point aveugle. Cet article a d'abord constitué une sorte de défi à relever face au quasi-néant et a fini par se transformer en un premier défrichage. S'il n'a encore nullement la prétention d'être exhaustif, ce recensement des textes des années 1840 et 1850 permet de valider certaines intuitions et d'ouvrir quelques pistes de réflexion.

La presse semblait offrir les meilleures chances de débusquer quelques textes dispersés, dont ceux déjà recensés par le collectif La vie littéraire au Québec. Je m'attarderai plus particulièrement au journal La Ruche littéraire qui, à l'instar de certains journaux parus précédemment, s'est efforcé de donner une place plus grande aux femmes et au féminin dans ses pages. Parmi les textes conservés dans la sphère privée, il existe également une pratique émergente qui a retenu notre attention et qui offre une étroite parenté avec la presse: les journaux littéraires de couventines. Il s'agira donc

5 Ma thèse explorait justement cet aspect: Stratégies épistolaires et écritures féminines: les Canadiennes à la conquête des lettres (1639-1839), thèse de doctorat (études littéraires), Montréal, Université du Québec à Montréal, 2002, [En ligne], [http://archipel.uqam.ca/id/eprint/9887]. Voir, également, Julie Roy, "Des réseaux en convergence: les espaces de la sociabilité littéraire au féminin au tournant du XIX siècle», Globe: revue internationale d'études québécoises, vol. 7, $\mathrm{n}^{\circ} 1$ (2004), p. 79-105. 
d'explorer les conditions particulières d'énonciation qui se mettent en place au cours des décennies 1840 et 1850 et les mutations de l'imaginaire littéraire qui s'opèrent pour les femmes et permettront l'émergence de vocations littéraires au cours des décennies subséquentes.

\section{La presse des années 1840}

Avec la publication remarquée de trois poésies et de trois récits dans Le Populaire en 1837-1838, on se serait attendu à ce que la plus illustre des femmes de lettres de la première moitié du XIX ${ }^{\mathrm{e}}$ siècle, Odile Cherrier, devienne l'une des auteures phares de sa génération et poursuive sa carrière littéraire grâce au soutien des éditeurs du Populaire, Joseph-Guillaume Barthe et Hyacinthe Leblanc de Marconnay, et de ses frères qui œuvraient alors dans le monde de la presse. L'éclatement des rébellions et le changement d'allégeance politique du Populaire semblent toutefois avoir eu raison de sa carrière littéraire, qu'elle laisse subitement en plan au début de l'année 1838. Odile Cherrier deviendra malgré tout une sorte de modèle d'acceptabilité pour celles qui suivront ses traces au début des années 1840. Tirant profit de son apprentissage de la bienséance et de l'art épistolaire, Odile Cherrier a bien retenu ses leçons de savoir-vivre lorsqu'elle écrit aux éditeurs du Populaire pour s'excuser de prendre la plume. Dans L'Aurore des Canadas, Améla et Albine copieront d'ailleurs toutes deux, mot pour mot, une phrase clé de la lettre d'introduction d'Anaïs dans laquelle elle demandait aux éditeurs du Populaire de bien vouloir publier son premier essai de littérature: "Je réclame avant tout cette bienveillance que vous avez pour tous les commençans [sic] et que réclame toujours, avec beaucoup plus d'espoir, le sexe auquel je me fais gloire d'appartenir ${ }^{6}$.»

6 Albine, «Mr. L'éditeur", L'Aurore des Canadas, 28 janvier 1840, p. 1-2. La lettre envoyée par Anaïs se lisait comme suit: "Il est certain que l'élève doit perdre à côté d'une semblable institutrice et c'est cette appréhension qui me porte d'avance à solliciter toute l'indulgence du public. Je ne me crois pas encore assez 
Jeunesse, inexpérience et demande de bienveillance, voilà trois éléments essentiels qui caractériseront la posture des femmes dans la presse au tournant des années 1840. Ces caractéristiques se retrouvent également chez Améla, qui rédige et fait paraître des textes qui se situent résolument dans le registre romantique qu'avait suivi Anaïs. L'absence de l'être cher, forte thématique de la correspondance féminine fictive, marque également le discours. De l'absence du frère bien aimé, envoyé au collège, pour Anaïs ${ }^{7}$ (et qui rappelle l'importance du couple frère et souur de la littérature romantique des années 1820 en France), l'absent devient l'amoureux exilé de force par les autorités coloniales après les rébellions. Les trois textes ${ }^{8} \mathrm{~d}^{1}$ Améla publiés en 1839-1840 décrivent la perte de l'être cher et sont destinés à attendrir le cœur certes, mais ils présentent également un enjeu politique. Cet amoureux, Pierre Damour, mort en exil des fièvres jaunes, était en effet bien connu pour avoir été l'un des grands agitateurs patriotes à Montréal, lui qui avait été dénoncé le 27 août 1837 par Le Populaire avant de gagner la Louisiane quelques mois plus tard. Le quatrième texte d'Améla intitulé "Mes souvenirs ou Améla sur la tombe de sa mère», paru le 23 octobre 1840 , vient mettre un terme à ses élans poétiques.

Dans un tout autre registre, «Albine», de Montréal, demande aux éditeurs de L'Aurore des Canadas de faire paraître un court texte qu'elle a rédigé et qu'elle juge pouvoir être utile à ses amies. Ce texte

forte pour me permettre de donner quelque chose de ma propre imagination; mais comme ma maxime est d'avoir un début, en quelque matière que ce soit, je réclame de vous cette bienveillance que vous avez pour tous vos commençans et que réclame toujours, avec beaucoup plus d'espoir, le sexe auquel je me fais gloire d'appartenir. ", Le Populaire, 25 octobre 1837, p. 1.

7 «L'amitié (À mon frère)», Le Populaire, 12 février 1838, p. 1.

8 Améla, "Stances sur la mort de Pierre G. Damour, médecin", L’Aurore des Canadas, 11 octobre 1839, p. 1; "Stances", L'Aurore des Canadas, 12 novembre 1839, p. 1; "Qui s'intéresse à ma mélancolie? ", L'Aurore des Canadas, 6 décembre 1839 , p. 1 ; "Mes souvenirs ou Améla sur la tombe de sa mère", L’Aurore des Canadas, 23 octobre 1840, p. 1. 
hybride est publié le 28 janvier 1840 et rappelle les lettres d'opinion parues sous pseudonymes féminins au cours des années 1820 et 1830 dans la presse québécoise. En toute naïveté, Albine mentionne à l'éditeur avoir traduit un texte signé "An old Maid» paru dans un journal anglais, au lieu de se concentrer sur sa leçon de musique. Elle ne publie pas ici une traduction comme l'avait fait Anaïs avec "Rosalie Berton» et "Une scène à St Domingue ${ }^{9}$ ». Cette mise en scène sert plutôt d'élément déclencheur puisque lorsqu'arrive son professeur de musique, celui-ci reste perplexe devant la traduction qu'elle lui propose de lire. Il la qualifie de "passable» et «moins que bonne» et suggère à sa jeune élève de laisser tomber la difficile technique de la traduction, qu'elle ne saurait maîtriser par ailleurs, pour se contenter de partager son opinion à propos des idées évoquées dans l'article. L'intention du texte d'Albine demeure ambiguë, mais ce dernier comporte plusieurs éléments d'intérêt permettant de saisir divers aspects de la posture de la collaboratrice de la presse pendant cette période et les diverses étapes menant de l'inspiration à la publication. Sur le plan de l'énonciation, le professeur n'est qu'un accessoire qui autorise Albine à prendre la parole. Le paratexte éditorial, qui consiste en une lettre aux éditeurs de L'Aurore des Canadas, lui permet de demander à nouveau l'autorisation de partager cette opinion, cette fois avec le public de la presse. En définitive, Albine, qui croit que ses amies qui se laissent facilement séduire par les jeunes hommes pourraient tirer grand profit de la lecture de ce texte, passe plus de temps à justifier sa prise de parole dans la presse qu'à la prendre réellement. Ce texte agit comme une sorte de métaphore des difficultés qu'éprouvent les femmes à prendre la parole publiquement.

Si l'on élimine les poésies "À une étoile tombante» et "À l'oiseau blanc» signées Josephte, parues dans la Revue canadienne en 1845 , et qui, on le sait maintenant, sont de Pierre-Joseph-Olivier

9 Anaïs, «Rosalie Berton. Trad. du Lady's Books de Philadelphie», Le Populaire, 25 octobre 1837, p. 1-2 et "Horrible tragédie: une scène à St-Domingue. Traduit de l'anglais ", Le Populaire, 17 janvier 1838, p. 1-2. 
Chauveau ${ }^{10}$, on doit attendre la toute fin de la décennie 1840 pour voir à nouveau quelques signatures féminines dans la presse. Ce sont encore deux poésies ayant pour thème la souffrance des femmes face à l'exil de leurs proches, qui paraissent le 31 octobre 1848 sous la plume de Betsy D. dans L'Aurore des Canadas. Intitulés "Le départ d'un amant. À Joseph Lartie» et "Le départ d'un amant. Pour Joseph Lartie», les deux poèmes s'adressent à Joseph Lartie, qui répondra en vers la semaine suivante ${ }^{11}$. Ces textes rappellent les poésies d'Améla sur la mort de Pierre Damour ${ }^{12}$. On le voit, la mort et l'exil des patriotes ont fortement marqué les esprits. Or, le sort des femmes qui restent et pleurent les absents est une thématique importante de la littérature épistolaire et de la correspondance intime qu'on voit ici transposé dans le discours poétique.

On trouve encore une poésie de Sophie B., de Les Cèdres en banlieue de Montréal, intitulée "La voix d'une mère ". Le texte paraît le 28 mars 1849 dans L'Avenir, un journal fondé par des membres de l'Institut canadien ${ }^{13}$. Ces conseils d'une mère à sa fille semblent presque venir expliquer l'absence des femmes dans la presse francophone au cours de la décennie 1840:

${ }_{10}$ "À une étoile tombante», Revue canadienne, 29 mars 1845, p. 113; "À l'oiseau blanc», Revue canadienne, 12 avril 1845, p. 137. Voir Lucie Robert, "Étranger à son temps et à lui-même" : l'écrivain et ses signatures ", Voix et Images, vol. 30, $\mathrm{n}^{\circ} 1$ (2004), p. 31-46.

${ }^{11}$ Betsy D., "Le départ d'un amant. À Joseph Lartie» et "Le départ d'un amant. Pour Joseph Lartie», L'Aurore des Canadas, 31 octobre 1848, p. 1; Larttie, "Pour Betsy. Romance", L'Aurore des Canadas, 7 novembre 1848, p. 1.

${ }^{12}$ Il est possible que Barthe soit le cerveau derrière Améla et Betsy D. Après avoir quitté Le Populaire pour des raisons idéologiques, il était devenu le rédacteur de L'Aurore des Canadas. Barthe avait notamment fait paraître un poème sur les exilés dans Le Fantasque de Napoléon Aubin, ce qui lui valut de se retrouver en prison. Notons aussi que Pierre Damour est un cousin d'Odile et d'AndréRomuald Cherrier.

${ }^{13}$ Parmi les fondateurs, on trouve Jean-Baptiste-Éric Dorion, Joseph Papin, Rodolphe Laflamme, Joseph Doutre, Charles Daoust, Wilfrid Dorion, Louis Labrèche-Viger et Charles Laberge. Voir Michel Lefèbvre, «Le journal L'Avenir (1847-1852)", Mémoires du livre, vol. 3, no 1 (automne 2011), [En ligne], [http://id.erudit.org/iderudit/1007574ar] (16 décembre 2019). 
Laisse à l'homme la gloire,

Les triomphes, le bruit,

Pour nous, aimer et croire

Au bonheur nous conduit.

Coule une vie obscure

Que le devoir remplit;

L'onde à l'ombre est plus pure,

Rien ne trouble son lit $^{14}$.

Un peu ironique sous la plume d'une femme qui s'adresse à la presse, il rappelle néanmoins le poème " $\left[\mathrm{Si}\right.$ tout votre avenir se fonde $\left.{ }^{15}\right]$ " que Louise-Amélie Panet Berczy avait offert à la jeune Marianne Stuart pour son album à l'été 1841 et qui visait à la mettre en garde contre les illusions de la gloire. Louise-Amélie Panet Berczy, on le sait, refusera de publier ses œuvres, pourtant appréciées de plusieurs lettrés. La décennie 1840 semble ainsi se terminer comme elle a commencé, les femmes restant marginales dans la presse de cette période. Même les lettres d'opinion qui ont marqué les années 1810-1820 semblent avoir été délaissées comme forme d'intervention publique au profit d'un certain repli dans la sphère privée.

\section{La presse des années 1850}

Dans La Minerve du 7 octobre 1852, journal dirigé par Ludger Duvernay, on trouve un poème de Caroline Desbois, de Laprairie, intitulé "Malheur ${ }^{16}$ ». Ce poème aborde le difficile passage de l'enfance à l'âge adulte et la douloureuse perte des illusions qui en résulte; une thématique qui était déjà présente dans plusieurs textes signés sous pseudonymes féminins depuis les débuts de la presse. C'est encore avec un texte rappelant les conséquences des rébellions qu’une

\footnotetext{
${ }^{14}$ Sophie B., "La voix d'une mère», L'Avenir, 28 mars 1849, p. 1.

${ }^{15}$ Louise-Amélie Panet, "[Si tout votre avenir se fonde] ", collection Baby, Archives de l'Université de Montréal, P58, boîte 95, pièces diverses, Q1/162, été 1841.

${ }^{16}$ Caroline Desbois, "Malheur», La Minerve, 7 octobre 1852, p. 2. "À peine touchons-nous au terme de l'enfance; Que déjà sous les pleurs et les chagrins amers; L'âme se courbe ainsi que l'arbrisseau se penche, Au souffle des hivers ».
} 
voix féminine se fait entendre sous le pseudonyme de Virginie $L^{*}$ à la fin de la décennie. Intitulé «Épitaphe pour le Monument consacré aux Victimes de 1837-1838", ce texte paraît d'abord dans La Patrie du 14 février $1858^{17}$. Ce journal montréalais (à ne pas confondre avec La Patrie d'Honoré Beaugrand) avait été fondé pour appuyer la coalition libérale-conservatrice de 1854, alors que l'érection d'un monument consacré aux patriotes soulevait toutes les passions. Les dissensions entre les libéraux de l'Institut canadien et le clergé conservateur étaient vives quant à l'inscription qui devait y être apposée. Le texte de Virginie L* est patriotique, mais il s'éloigne des querelles politiques pour leur préférer la compassion à l'égard de ces hommes qui ont sacrifié leur vie pour la patrie et pour leurs familles.

En clôture de la décennie 1850, on trouve encore dans le Journal de l'instruction publique un texte qui aurait bien pu passer inaperçu. Au mois de novembre 1858, le rédacteur, Pierre-JosephOlivier Chauveau, y publie le poème "L'été de la Saint-Martin ${ }^{18}$ ». En note, il indique que « [c] ette charmante description est empruntée à l'album d'une dame canadienne qui, à notre grand regret, ne veut pas même permettre que nous indiquions ses initiales ${ }^{19}$ ". Ce poème, on l'apprendra dans le numéro du mois de mai $1862^{20}$, est en fait un extrait du long poème "Les saisons du Bas-Canada" qu'elle avait offert à Jacques Viger pour son album Souvenirs canadiens en $1838^{21}$. Écrit vingt ans plus tôt, il s'inscrit dans la veine

${ }_{17}$ Virginie L*, "Épitaphe pour le Monument consacré aux Victimes de 18371838 ", La Patrie, 14 février 1858, p. 2.

${ }^{18}$ Ce poème a été édité par Roger Le Moine, dans Louise-Amélie Panet, Quelques traits particuliers aux saisons du Bas Canada et aux mours de l'habitant de ses campagnes il y a quelque quarante ans, Orléans, Éditions David, 2000, coll. "Voix retrouvées".

${ }^{19}$ Journal de l'instruction publique, Montréal, vol. 2, n 11 (novembre 1858), p. 198.

${ }^{20}$ Journal de l'instruction publique, Montréal, vol. 6, n 2 (mai 1862), p. 102.

${ }^{21}$ Grâce à une note de Chauveau laissée dans l'album en date du 27 juin 1856, on peut supposer que l'album dont il parle alors est celui aujourd'hui connu sous le nom d'Album Jacques Viger: Souvenirs canadiens. 
romantique du début $\mathrm{du} \mathrm{XIX}^{\mathrm{e}}$ siècle, mais annonce également cette idée d'une littérature "canadienne" qui s'épanouira dans les années 1860 .

\section{Le bourdonnement des abeilles de La Ruche littéraire}

Le journal La Ruche littéraire, publié à Montréal à partir du mois de mars 1853, détonne dans le paysage médiatique de ces deux décennies à plusieurs titres. En apparence moins politique que la plupart des journaux de la période, il mise sur l'ouverture à la littérature européenne et américaine et fait montre d'un intérêt soutenu pour le lectorat féminin. Si d'autres journaux, surtout à partir des années 1860, s'adressent aux familles et donc aux mères de famille, La Ruche littéraire va déjà nettement plus loin. L'éditeur GeorgesHippolyte Cherrier (le frère d'Odile Cherrier, qui sera d'ailleurs agente de La Ruche littéraire) et son rédacteur, Henri-Émile Chevalier, ont pour objectif de créer un journal qui fera rayonner la littérature canadienne. Dès le second numéro, ils souhaitent porter une attention particulière à leurs lectrices:

Afin de nous gagner le patronage des dames, - patrons auxquels soit dit en passant nous tenons infiniment, - nous joindrons fréquemment à nos Tablettes éditoriales, un article de MODES, dû à la plume d'une Parisienne bien connue pour l'élégance de sa toilette, et la coquetterie de son style ${ }^{22}$.

Même les romans et chroniques choisis par La Ruche proviennent d'auteures connues en Europe et aux États-Unis. On trouve évidemment plusieurs textes de femmes de lettres françaises dans la presse québécoise (surtout des poètes du XVIII ${ }^{\mathrm{e}}$ siècle et du début du $\mathrm{XIX}^{\mathrm{e}}$ siècle), mais La Ruche fait une large place aux auteures contemporaines. Le roman d'Harriet Beecher Stowe, Uncle Tom's Cabin, paru aux États-Unis en 1852 et qui connaît un succès populaire sans précédent, en est un bon exemple. La traduction d'Émile de la

${ }^{22}$ La Ruche littéraire illustrée, vol. 1, nº 2 (mars 1853), p. 44. 
Bédollière, parue à Paris sous le titre La case du père Tom au début de l'année suivante, sera au cour de La Ruche pendant plusieurs mois, témoignant à la fois de l'ouverture de Chevalier à l'écriture des femmes, de son goût pour la production contemporaine, mais aussi de la solidité de son réseau qui lui permet d'y avoir accès rapidement $^{23}$. La Ruche publie également des textes destinés à parfaire les connaissances des femmes, comme la chronique d'agronomie intitulée «De l'éducation agricole des femmes» de Frédéric Ossaye ${ }^{24}$. Chevalier s'engage même auprès de ses lectrices à tenir compte de leurs suggestions ${ }^{25}$.

Mais le rédacteur souhaite bien plus de la part de ses lectrices:

Cependant nous ne serions pas fâchés non plus qu'elles ne s'en tinssent pas uniquement à des avis et qu'elles nous fissent l'honneur de nous adresser de ces délicieuses bluettes, dont elles seules possèdent le secret. Dans les publications anglaises, nous voyons, fréquemment, figurer le nom d'une charmante Miss ou d'une ravissante Mrs; pourquoi donc n'en serait-il pas de même dans les publications françaises? Est-ce que, d'aventure, nos dames ou demoiselles auraient plus profonde aversion de l'encre que leurs rivales d'Amérique ou de la Grande-Bretagne? Il est vrai qu'un doigt blanc, maculé de noir, peut dégoûter le vulgaire: mais l'homme de génie, remarquant le cachet de sa nature, s'incline et maudit le savon chargé d'effacer ce signe de l'intelligence, une femme auteur, c'est un trésor pour le public ${ }^{26}$.

${ }^{23}$ Pour une histoire de sa traduction française, voir Claire Parfait, "Un succès américain en France: La case de l'oncle Tom», E-rea, 7,2, 2010, [En ligne], [http:// erea.revues.org/981] (16 décembre 2019).

${ }^{24}$ La Ruche littéraire illustrée, vol. 1, n 4 (mai 1853), p. 210.

25 «Plusieurs femmes d'esprit, et elles sont nombreuses en Canada, ont daigné nous écrire pour nous indiquer le genre de littérature qui leur paraît le plus propre à assurer la réussite de notre œuvre; nous remercions bien sincèrement ces dames de la part qu'elles veulent prendre à nos succès futurs, et nous leur promettons fidèle observance à des conseils dictés avec le désintéressement habituel du beau sexe» (La Ruche littéraire et illustrée, vol. 1, no 4 [mai 1853], p. 230).

26 "Tablettes éditoriales», La Ruche littéraire illustrée, vol. 1, n 4 (mai 1853), p. 230. 
La lectrice attentive aura remarqué que la section contenant les remerciements aux dames canadiennes pour leurs conseils littéraires est intitulée "Piège-à-Dames" et que l'éloge de ce "trésor pour le public» qu'est "une femme auteur» reconduit les éternels lieux communs de la supériorité littéraire des femmes et a pour titre «Perfide apologie du beau sexe». Le rédacteur, malgré ses penchants favorables à l'écriture des femmes, est néanmoins conscient des dangers qui guettent celles qui se lancent en littérature. C'est pourquoi il joue la carte du double discours. En avril 1853, Chevalier recommande chaudement la lecture du Snow Drop, un journal pour enfants produit à Montréal par Elizabeth Lanesford Cushing et Harriet Vaughan Cheney entre avril 1847 et juin 1853. On remarque le ton fleur bleue et la surenchère de clichés:

C'est une de ces rares et délicieuses productions qui font doucement rêver et inondent le cœur des plus tendres sensations. Aussi ne saurions-nous trop remercier Mesdames Cushing et Cheney du plaisir qu'elles nous ont procuré en nous envoyant un échantillon de la dernière corbeille de fleurs qu'elles viennent exposer à l'admiration publique. Nos jeunes amies, à qui elle est spécialement dédiée, y trouveront de suaves primevères, des fruits savoureux et comme nous butineront $[s i c]$ au sein de ces fraîches primeurs avec une allégresse, qui comme nous également, les fera soupirer pour une nouvelle apparition du Snow Drop ${ }^{27}$.

Ce texte rappelle les discours des éditeurs sur l'écriture des femmes. On n'a qu'à relire les textes de Hyacinthe Leblanc de Marconnay et de Joseph-Guillaume Barthe à propos des écrits de Marie-Louise et d'Anaïs parus dans Le Populaire 20 ans plus tôt pour s'en convaincre.

Quant à la chronique de mode promise aux lectrices dans le premier numéro de La Ruche, elle sera tenue par Rosalie $M^{* * *}$, une soi-disant «Française» qui ne manque pas de vivacité :

Soyez satisfait, riez complaisamment dans votre barbe, vous m’avez clouée à mon pupitre, j'ai la sottise de répondre à votre

${ }^{27}$ La Ruche littéraire illustrée, vol. 1, nº 3 (avril 1853), p. 172. 
inqualifiable demande. Mais..... j'aurai ma revanche. Vous vous souviendrez du mauvais tour que vous me jouez! Ah! vous vous figurez qu'il ne s'agissait que de dire «ma chère, je veux un article de modes parisiennes et je compte sur vous» pour être servi! Eh bien, monsieur le mal-appris, je refuse, moi, de vous obéir, à moins que vous ne me fassiez le serment solennel de publier, mensuellement, dans la Ruche Littéraire, un article de modes canadiennes ${ }^{28}$.

Même si La Ruche ne fera paraître qu'une seule chronique de «modes montréalaises», Rosalie $\mathrm{M}^{* * *}$ signera néanmoins onze chroniques entre juin 1853 et septembre 1854. Lorsque les lettres de Rosalie $\mathrm{M}^{* * *}$ tardent à arriver, Chevalier publie des chroniques de Julie de Marguerittes ${ }^{29}$ et de la vicomtesse de Renneville ${ }^{30}$, deux chroniqueuses connues en France et aux États-Unis.

Dans le numéro de mai 1854, le journal annonce encore la venue d'une nouvelle collaboratrice: "Nous avons le plaisir d'annoncer à nos lecteurs que $L a$ Ruche s'est acquis la précieuse collaboration de Mme Manoël de Grandfort ${ }^{31}$ ». Suivra une longue biographie de l'auteure, sous la rubrique "Célébrités contemporaines», ainsi que la reproduction, en note, d'un compte rendu de son passage comme conférencière à l'Institut canadien de Montréal et la remise d'une coupe d'argent sur laquelle était inscrit: «Hommage au génie de la femme ${ }^{32}$ ».

${ }^{28}$ Rosalie $\mathrm{M}^{* * *}$, «Modes», La Ruche littéraire illustrée, vol. 1, no 5 (juin 1853), p. 298.

${ }^{29}$ « $\mathrm{M}^{\mathrm{me}}$ la baronne de Marguerittes et $\mathrm{M}$. Arpin ancien rédacteur-en-chef du Courrier des États-Unis, se sont engagés à nous donner chaque mois des Chroniques ou Revues de New-York. Cette brillante collaboration va placer la Ruche à la hauteur des publications littéraires les plus estimées ", La Ruche littéraire illustrée, vol. 1, n 5 (juin 1853), p. 297. Julie de Marguerittes est une Italienne, mariée à un baron français, exilée à New York, coéditrice du journal The Verdict et auteure polygraphe.

${ }^{30}$ Pseudonyme d'Olympe Vallée, $\mathrm{M}^{\mathrm{me}}$ Paul Descubes de Lascaux est une chroniqueuse de mode française prolifique.

${ }^{31}$ La Ruche littéraire et politique, vol. 3, no 4 (mai 1854), p. 2.

${ }^{32}$ H. E. C., "Marie-Antoinette Barsalou ( $\mathrm{M}^{\mathrm{me}}$ Manoël de Grandfort)», La Ruche littéraire et politique, vol. 3, no 5 (juin 1854), p. 297-302. 
C'est toutefois dans ses "Tablettes éditoriales» que Chevalier laisse entrevoir ce qui se trame dans la salle de rédaction et ses échanges avec de nouvelles collaboratrices venues des quatre coins de l'Amérique. Le "Rêve d'une esclave» de $\mathrm{M}^{\mathrm{me}}$ Elvira de la NouvelleOrléans est refusé pour des raisons éthiques, le texte de $\mathrm{M}^{\mathrm{me}}$ Octavie ne peut être publié car il contient trop d'erreurs grammaticales. Quant au texte de $M^{\text {lle }}$ L. D. de Trois-Rivières, il ne sera pas publié, malgré ses qualités, puisque le journal n'accepte aucune communication sans nom d'auteur. En juin 1853, on apprend encore que Chevalier a reçu le poème "Rêves d'amour» de Malvina $\mathrm{D}^{* * *}$ de Québec: «[C]ette suave aspiration d'un cœur sensible, - la première dont une de nos aimables Canadiennes ait daigné nous honorer $[\ldots]^{33}{ }$. Ce premier poème, publié dans la livraison de juillet, met en scène un jeune homme dénonçant l'inconstance de sa bien-aimée. Chevalier invite alors les dames de Québec à suivre l'exemple de Malvina $\mathrm{D}^{* * *}$ : «Puisse la contagion exercer des ravages parmi le beau sexe de Québec ${ }^{34}$.»

S’il y eut, semble-t-il, peu de "contagion" parmi les Québécoises, Malvina $\mathrm{D}^{* * *}$ sera bien davantage qu'une collaboratrice momentanée. En novembre 1853, elle adresse à La Ruche un texte intitulé "Gazouillement ${ }^{35}$ ». Il s'agit du récit de la rencontre fortuite d'une jeune femme qui, après avoir écarté la vie religieuse de ses aspirations et décidé que le mariage ne lui convenait pas, avait choisi le célibat. Malgré ses convictions, elle raconte à la narratrice le piège tendu par un jeune homme à qui elle avait ouvert son cœur. Après qu'elle eut cédé à ses avances, ce dernier l'avait lâchement abandonnée, et la jeune femme brisée vivait maintenant retirée du monde à la campagne. La narratrice, qui s'était assoupie, se réveille

33 "Tablettes éditoriales», La Ruche littéraire illustrée, vol. 1, n 5 (juin 1853), p. 297. "Rêves d'amour" paraît en juillet 1853, dans La Ruche littéraire illustrée, vol. $1, \mathrm{n}^{\circ} 6$, p. 3 .

${ }^{34}$ La Ruche littéraire illustrée, vol. 1, no 6 (juillet 1853), p. 3.

${ }^{35}$ Malvina $\mathrm{D}^{* * *}$, "Gazouillement", La Ruche littéraire et politique, vol. 2, $\mathrm{n}^{\circ} 4$ (novembre 1853), p. 578. 
ne sachant plus si la rencontre avait eu lieu ou s'il s'agissait d'un rêve. Dans les deux cas, cela devait certainement lui servir d'avertissement pour son propre avenir.

Un autre texte de Malvina $\mathrm{D}^{* * *}$, «De Québec à la chute de Montmorency ${ }^{36}{ }^{\prime}$, est publié en mai 1854 . Sous le prétexte d'une excursion sur la côte de Beaupré, le lieu touristique le plus prisé de la banlieue de Québec, le récit met en scène les échanges entre Alfred et son ami Ernest, un Français récemment installé à Québec. Les paysages sont grandioses, et Alfred ne peut s'empêcher de provoquer Ernest: «[D]ites-moi si [...] en perdant cette contrée, la France n’a pas perdu le plus beau joyau de sa couronne?», annonçant du coup le programme sous-jacent. La randonnée terminée, les deux hommes s'arrêtent dans une maison au pied des chutes Montmorency, avant de prendre le chemin du retour. C'est là que se produit la rencontre inopinée avec Marie. Débute alors la seconde partie du récit: l'histoire d'amour entre Alfred et Marie et de leur plan de mariage contrecarré par la volonté des parents de la jeune fille de la voir épouser un Anglais pour l'avancement de la famille. Succombant à la folie le soir même de ses noces, Marie sera internée à l'asile de Beauport. Elle s'en échappe ce jour-là, comme elle le faisait régulièrement, pour revoir l'endroit où Alfred et elle s'étaient promis fidélité. Au-delà du toast à la France qui souligne les regrets d'Alfred pour l'ancienne mère patrie, il est question ici encore, de manière transposée, des souffrances endurées par les femmes qui paient le prix des jeux politiques dont elles sont les innocentes victimes.

Quant au quatrième texte envoyé par Malvina $\mathrm{D}^{* * *}$ et intitulé "Promenade en bateau», il semble avoir fait les frais de la fermeture $\mathrm{du}$ journal en décembre 1854. Malvina $\mathrm{D}^{* * *}$ est non seulement le seul pseudonyme de permanence observé au cours des décennies 1840-1850, mais lorsque les noms des collaborateurs principaux apparaissent en page titre de La Ruche littéraire à partir du

${ }^{36}$ Malvina $\mathrm{D}^{* * *}$, «De Québec à la chute de Montmorency», La Ruche littéraire et politique, vol. 3, $\mathrm{n}^{\circ} 4$ (mai 1854), p. 220-226. 
mois de mars 1854 , le nom de Malvina $\mathrm{D}^{* * *}$ est inscrit bien en vue parmi ceux de collaborateurs connus comme Victor Baron, J. Gentil, Auger Delbreau, Félix Vogeli et... Rosalie $\mathrm{M}^{* * *}$. C'est une première dans l'histoire de la presse québécoise que de voir ainsi affichés des noms de femmes comme collaboratrices régulières. Il faudra attendre L'album de la Minerve en 1872 pour voir à nouveau des noms de femmes figurer parmi les membres réguliers de la rédaction d'un journal. Lors de la réouverture de La Ruche littéraire au printemps 1859 , Virginie $\mathrm{B}^{* * *}$ signe un poème intitulé "Croquis ${ }^{37}$ " dans lequel elle décrit ce qu'elle voit de sa fenêtre. Ce sera le dernier texte original signé sous pseudonyme féminin dans La Ruche littéraire.

Cette poignée de textes que nous venons de présenter ne révolutionne certainement pas l'histoire littéraire des femmes pour ce qui est du nombre. Nous sommes également en droit de nous demander qui se cachaient sous plusieurs de ces pseudonymes. Comme l'indiquent les éditeurs de La Revue canadienne lorsqu'ils acceptent de publier les deux poèmes de Pierre-Joseph-Olivier Chauveau signés Josephte en 1845: «Nous ne publions jamais un article sans en connaître l'auteur, mais nous faisons volontiers une exception en faveur des dames ${ }^{38}$." Émile Chevalier semble plus pointilleux à ce propos (rappelons-nous qu'il refuse de publier le texte de $\mathrm{M}^{\text {lle }} \mathrm{L}$. D. de Trois-Rivières parce qu'elle avait omis de s'identifier), mais il respectait néanmoins la volonté des collaborateurs de garder leur identité à l'abri des regards du public. On ne saura sans doute jamais qui se cachait derrière le pseudonyme de Malvina $\mathrm{D}^{* * *}$ ou de Virginie $\mathrm{B}^{* * *}$. On ne doit toutefois pas oublier qu'il est possible que des femmes aient utilisé l'anonymat, les initiales ou d'autres subterfuges, comme l'adoption d'un pseudonyme masculin, pour faire publier leurs œuvres sans

37 "Croquis. À M..., le 15 mars 1859 », La Ruche littéraire, vol. 5, n 2 (avril 1859), p. 50 .

${ }^{38}$ Revue canadienne, 29 mars 1845, p. 113. 
compromettre leur réputation. Nous avons observé l'utilisation de plusieurs de ces stratégies visant à mettre sa réputation personnelle à l'abri lors des décennies subséquentes. Le refus de Louise-Amélie Panet Berczy de laisser publier ses œuvres sous son propre nom nous rappelle cette pudeur, tout comme le récit que fait Azélie Papineau de ses démarches pour faire publier quelques chroniques musicales dans la Revue canadienne en $1868^{39}$. Elle n'en est pas à ses premières armes, et les prières qu'elle adresse à Dieu pour lui enlever ce goût de l'écriture "par n'importe quels moyens" sont typiques d'un certain discours touchant les travaux intellectuels, notamment dans les couvents où certaines religieuses déployaient leurs talents dans les matières scientifiques. Mais, dans le présent cas, ce ne sont pas tant les savoirs trop poussés qui éloignent de Dieu que les conséquences de la publication sur le fragile édifice de la réputation et de l'humilité féminine.

\section{Les couvents comme lieu d'émulation}

Si la crainte de la critique et la gloire de la presse semblent en avoir rebuté plusieurs, il faut sans doute regarder ailleurs pour trouver des traces de la vie littéraire des femmes au cours des décennies 18401850. Les couvents ont toujours été des espaces propices à l'activité intellectuelle et artistique des femmes, particulièrement au cours de ces deux décennies :

Il se faisait alors une grande réaction dans le pays à propos de l'introduction de l'instruction supérieure dans les maisons religieuses. Les supérieurs ecclésiastiques étaient à la tête de ce mouvement: notre communauté ne demeura pas en arrière. Elle

\footnotetext{
39 «Quelle affaire que d'écrire dans un journal: mon petit mystère m'a causé plus d'embarras et de peine que de plaisir [...]. Comme par le passé, je demande à Dieu de m'ôter cette envie d'écrire si elle ne doit pas concourir à sa gloire et à mon salut: qu'il m'en dégoûte par n'importe quels moyens! Et si je continue, je ferai passer cela par les mains de mon mari. "Voir Mylène Bédard, Écrire en temps d'insurrections: pratiques épistolaires et usages de la presse chez les femmes patriotes (1830-1840), Montréal, Les Presses de l'Université de Montréal, 2016, p. 285.
} 
envoya une des meilleures élèves qui désiraient entrer au noviciat terminer son éducation dans un institut des plus renommés des États-Unis ${ }^{40}$.

On assiste, en effet, à la multiplication des maisons religieuses vouées à l'enseignement, à l'établissement d'écoles sur un plus vaste territoire et à la mise en place de programmes scolaires enrichis. Les religieuses encouragent les élèves à développer leurs habiletés littéraires: "Les discussions historiques, les joutes littéraires créaient l'émulation parmi les élèves. La maitresse donnait l'initiative, encourageait les bonnes volontés, mais elle demandait un travail personnel et le Cahier d'Honneur de ces années conserve des pages d'un mérite réel ${ }^{41}$.» On assiste également à l'ouverture des séances d'examens de fin d'année au public. L'historienne des Ursulines considère d'ailleurs cet élément comme suffisamment remarquable pour y consacrer une longue description ${ }^{42}$. Bien que l'on trouve parfois des comptes rendus des séances d'examens et des visites de dignitaires dans la presse dès les années 1770, les articles qui y sont consacrés deviennent monnaie courante à partir du milieu du $\mathrm{XIX}^{\mathrm{e}}$ siècle seulement ${ }^{43}$. Le Journal de l'instruction publique, destiné principalement aux enseignants et visant à faire la promotion de l'éducation, publié à partir de 1857, sera un fervent promoteur de ces comptes rendus. C'est ainsi que de jeunes couventines voient leurs noms imprimés dans la presse comme récipiendaires de prix et, parfois, comme récitantes ou même auteures de textes lus lors des séances de fin d'année. Repris dans divers journaux, ces comptes

${ }^{40}$ Les Ursulines des Trois-Rivières depuis leur établissement jusqu'à nos jours, tome 2, Trois-Rivières, P. V. Ayotte libraire-éditeur, 1892, p. 383.

${ }^{41}$ Les Ursulines des Trois-Rivières depuis leur établissement jusqu'à nos jours, tome 3, Montréal, A. P. Pigeon imprimeur, 1898, p. 127.

${ }^{42}$ Mère Sainte-Marie, Les Ursulines de Québec depuis leur établissement jusqu'à nos jours, tome 4, Québec, C. Darveau, 1866, p. 732-733.

${ }^{43}$ Le premier texte signé par des femmes identifiées dans la presse francophone est un compliment des jeunes élèves de l'Hôpital général de Québec adressé à lord et lady Carleton et paru dans la Gazette de Québec en 1774. 
rendus contribuent à célébrer les talents littéraires féminins et à rendre la jeune fille instruite et lettrée non seulement acceptable, mais recherchée.

\section{L'Annuaire et Le Papillon littéraire}

Les journaux de couventines sont créés par les communautés religieuses enseignantes pour susciter l'émulation parmi leurs élèves. Ils nous permettent aujourd'hui non seulement d'accéder à un corpus de textes écrits par des auteures identifiables, mais d'étudier un phénomène sans précédent: des jeunes filles, en compagnie de leurs enseignantes, créent un périodique sous forme manuscrite à l'image de ceux qui circulent dans leurs familles, mais desquels les femmes semblent quasi absentes. Le premier exemple connu de ce type de "publication" émane des Ursulines de Trois-Rivières, en décembre 1845, sous le titre L'Annuaire. L'épigraphe de L'Annuaire, tirée apparemment d'une phrase de Voltaire: "La perfection marche lentement: il lui faut la main du temps", donne à lire qu'il s'agit bel et bien d'un champ de pratique vers la perfection morale certes, mais également littéraire. L'Annuaire comprend les compositions d'élèves recopiées par les plus douées en calligraphie ainsi que quelques dessins visant à illustrer ou à enjoliver le propos: "Le premier article intitulé Ma patrie est de Flavie Gervais ${ }^{44}$. La copiste est Bernardine Gagnon; son écriture est remarquablement belle et les dessins, réussis, sont de Mary Ann O’Neil ${ }^{45}$." Le journal comprend des morceaux d'histoire, de courts récits et de la poésie en français et en anglais puisque « [1]'anglais y était enseigné sur le même pied que le français ${ }^{46}$ ». On y trouve également des annonces d'activités qui animent le couvent, des comptes rendus

\footnotetext{
${ }^{44}$ Flavie Gervais (1823-1875) deviendra mère de Saint-Olivier chez les Ursulines de Trois-Rivières.

${ }^{45}$ Histoire des Ursulines des Trois-Rivières depuis leur établissement jusqu’à nos jours, tome 3, p. 64.

${ }^{46}$ Ibid., tome 3, p. 59.
} 
de fêtes, de retraites et de visites officielles, des annonces plus triviales comme les heures d'ouverture de la bibliothèque ou l'arrivée imminente de nouveaux livres, de fournitures de dessin et de couture.

Dix ans plus tard, un autre journal de couventines voit le jour chez les Ursulines de Québec. Le premier numéro du Papillon littéraire paraît en décembre 1855 . Les membres de la toute nouvelle société littéraire de Sainte-Ursule sont invitées à y proposer poésies, récits, biographies, comptes rendus, lettres, analyses littéraires et traductions, qu'elles disposent dans un cahier qui prend la forme d'un journal périodique manuscrit et dans lequel les noms des auteures sont bien en vue. Les productions sont lues et commentées par le chapelain des Ursulines, Louis-Georges Lemoine, qui, en tant que premier public et instigateur du projet, permet aux élèves de s'initier à la critique. Le "Bulletin politique» que le chapelain rédige pour chacun des numéros vise à informer les couventines des grands événements qui secouent la scène politique internationale ${ }^{47}$. On est d'ailleurs étonné de l'importance accordée à l'histoire et aux grands événements de l'actualité politique dans les pages du Papillon littéraire, des textes qui côtoient les œuvres d'histoire religieuse, de piété et de morale. Contrairement aux attentes bercées par les préjugés, les poésies légères ne composent pas l'essentiel de ce journal.

Les couventines doivent également prendre en compte un public qui dépasse le cercle restreint de la petite société littéraire

${ }^{47}$ Le chapelain Louis-Georges Lemoine avait fait part de ses loisirs de jeune collégien aux couventines. Ces loisirs consistaient à recréer une chambre d'assemblée où chaque élève jouait le rôle d'un député. Il les avait invitées à trouver une façon équivalente, mais plus adaptée à une jeune fille, de contrer l'oisiveté. Les journaux qui voient le jour dans les collèges masculins visent sans doute les mêmes objectifs, mais se dotent de moyens différents. En effet, les journaux de collège sont souvent imprimés, et les collégiens ont la possibilité de collaborer aux entreprises de presse établies. Voir Léon Debien, "Les journaux de collège au XIx ${ }^{e}$ siècle", Mens: revue d'histoire intellectuelle de l'Amérique française, vol. 5, $\mathrm{n}^{\circ} 2$ (printemps 2005), p. 345-395. 
puisque toutes les élèves du couvent sont invitées à assister aux séances pendant lesquelles le journal est lu tandis que les parents, amis et dignitaires sont invités à le feuilleter lors de leurs visites. Le Papillon littéraire sera un outil privilégié de la formation littéraire des couventines jusqu'en $1864^{48}$. Chaque année, une douzaine de jeunes filles des classes supérieures ont ainsi composé, lu, relu, corrigé, transcrit et mis en pages Le Papillon littéraire. Pensée et conçue comme un véritable périodique, cette publication n'est pas qu'un loisir sans conséquence, mais bien un véritable lieu d'appropriation et de légitimation de la pratique littéraire publique.

\section{Conclusion}

Le nombre restreint de textes signés sous pseudonymes féminins recensés dans la presse ne révolutionne certes pas l'image de la participation des femmes à la vie littéraire au cours des décennies 1840-1850. On constate néanmoins certains changements, qui témoignent d'une forme de transition dans les pratiques jusque-là observées. Contrairement à la majorité des textes parus dans la presse avant 1840, qui entretenaient un certain dialogue avec la figure d'autorité de l'éditeur, soit par une lettre d'accompagnement ou une série de justificatifs, les poésies, qui constituent désormais le genre le plus prisé, ne semblent plus s'encombrer de ces lettres cherchant à obtenir la bienveillance des éditeurs et du public. Ces lettres à l'éditeur continuent sans doute à servir de vecteur pour rejoindre la rédaction des journaux, mais elles tendent à être éliminées du paratexte éditorial, laissant sous-entendre que cette mise en scène n'est plus nécessaire. C'est une transformation importante des pratiques éditoriales qui modifiera du même coup l'imaginaire entourant la création littéraire des femmes et qui témoigne de son acceptabilité croissante.

${ }^{48}$ Après une pause de quatre années, la société littéraire se restructure pour devenir le "Cercle littéraire de Sainte-Angèle». En 1868, Le Papillon littéraire devient alors L'Écho du cloître, jusqu'à l'arrêt définitif en 1891. 
Les injonctions quant aux genres, aux sujets et aux types de littérature auxquels les femmes pouvaient s'adonner ont certainement eu une influence sur le choix de prendre la parole ou non publiquement et surtout sur les conditions dans lesquelles elles ont pris cette parole. Difficile à distance et par manque de témoignages directs de décider si le peu de textes écrits par des femmes est le résultat d'un rejet de la part des éditeurs ou la conséquence d'une forme d'autocensure exercée par les femmes elles-mêmes. Les limites sont d'ailleurs généralement bien exposées par les éditeurs qui accueillent les textes signés par des femmes. On les détecte aisément dans la peine qu'ils se donnent à encenser les qualités dites féminines des œuvres qu'ils prennent le soin de publier. C'était évident dans les propos de Hyacinthe Leblanc de Marconnay au sujet des écrits de Marie-Louise et d'Odile Cherrier dans Le Populaire. Ça l'est encore dans La Ruche littéraire lorsqu'Émile Chevalier fait abondamment appel aux stéréotypes pour définir ce que devrait être l'écriture féminine. Néanmoins, si la plupart des textes publiés sous pseudonymes féminins au cours de ces deux décennies se plient à ces modèles, d'autres y échappent.

Quant à l'influence des journaux de couventines sur la participation des femmes à la presse, elle se révèle, bien sûr, difficilement quantifiable. La mise en place de sociétés littéraires et la création de journaux comme L'Annuaire et Le Papillon littéraire ont toutefois sans aucun doute contribué à développer chez les jeunes filles les dispositions nécessaires à la pratique de l'activité littéraire. Ce ne sont évidemment pas toutes les jeunes filles initiées à la littérature dans les couvents qui deviendront auteures ou journalistes par la suite, mais il est certain que cette forme de préparation au travail littéraire et journalistique a contribué à la création d'un habitus et influencé l'imaginaire entourant la femme de lettres et la femme journaliste ${ }^{49}$.

${ }^{49}$ Voir Julie Roy et Chantal Savoie, «De la couventine à la débutante: signature féminine et mise en scène de soi dans la presse au XIX ${ }^{e}$ siècle», Actes du colloque international «La lettre et la presse: poétique de l'intime et culture médiatique», 
Elle a aussi inévitablement contribué à définir les conditions et les modalités qui allaient autoriser l'émergence de pratiques d'écriture publiques, mais surtout à rendre acceptable cette forme d'investissement personnel dans les travaux littéraires. Avant de se faire connaître sous le nom de Laure Conan, Félicité Angers, alors jeune couventine, a fait ses premières armes dans Le Papillon littéraire dans les années 1860. Plusieurs jeunes filles éduquées dans les couvents des Ursulines au cours de ces deux décennies ont contribué à la vie littéraire et culturelle de leur temps.

Ces deux décennies sont également marquées par la parution du Répertoire national ou Recueil de littérature canadienne de James Huston, qui constitue la première compilation de textes littéraires canadiens. Dans la publicité visant à faire connaître cette nouvelle parution, on peut lire les noms de quelques auteurs dont celui d'Anaïs, auteure de la nouvelle "Une scène à Saint-Domingue 50 ". On doit également noter que la majorité des textes féminins recensés pendant cette période sont réédités, soit dans le même journal à une date ultérieure ou par des journaux concurrents. Le poème "La voix d'une mère" de Sophie B. est publié à nouveau dans L'Écho des campagnes du 19 avril 184951, et le poème "Malheurs» de Caroline Desbois est repris dans L'Ere nouvelle du 15 décembre $1852^{52}$. "L'épitaphe ${ }^{53}$ ", de Virginie $L^{* * *}$, sera reprise, quant à elle, dans La Patrie du 20 février 1858, puis rééditée dans La Guêpe, le 3 mai 1859, et dans Le Littérateur canadien du 3 juillet 1860. Ce phénomène a sans doute contribué à faire connaître ces textes à un plus large public et à donner une plus

Québec, mai 2010, Médias 19, [En ligne], [http://www.medias19.org/index. php?id=318] (16 décembre 2019).

${ }^{50}$ James Huston, Le répertoire national, tome 2, Montréal, Imprimerie de Lovell et Gibson, 1848, p. 114-118.

${ }^{51}$ L'Écho des campagnes, 19 avril 1849, p. 1.

52 L'Ére nouvelle, 15 décembre 1852, p. 1.

${ }^{53}$ La Patrie, 20 février 1858, p. 333; La Guêpe, 3 mai 1859, p. 1 et dans Le Littérateur canadien ( $\left.\mathrm{L}^{* * *}\right), 3$ juillet 1860 , p. 5. 
grande visibilité à la littérature produite par des Canadiennes ou assumée telle.

La création d'un habitus littéraire qui permettra aux Canadiennes de prendre leur place sur la scène publique semble s'être nourrie à la pratique littéraire des couvents, tout autant qu'aux représentations collectives qui, graduellement, $s^{\prime}$ inscrivent dans l'espace public. Comme l'indique Jérôme Meizoz, l'auteur, en dépit de sa quête d'originalité, puise obligatoirement "à un répertoire présent dans la mémoire des pratiques littéraires ${ }^{54}$ " pour se construire une "posture». Qu'ils proviennent de femmes réelles ou de femmes fictives, de la presse ou des couvents, ou même d'exemples de femmes de lettres étrangères, ces divers modèles ont contribué à baliser la conquête de la sphère littéraire et journalistique qu'amorceront les femmes au cours des décennies suivantes. Comme le note Daniel Mativat au sujet de cette période de l'histoire littéraire, «[1]'écrivain des années 1850-1860 commence à découvrir la spécificité de l'activité littéraire et, même si on ne peut encore parler de conscience professionnelle et de recherche d'une véritable autonomie, il ne fait pas de doute que les mentalités sont en train de changer ${ }^{55}$ ». Bien que tout ne soit pas encore en place pour propulser les femmes vers la pratique littéraire publique, la période $1840-1860$ a permis aux femmes de faire graduellement leur place et de préparer l'éclosion qui marquera les années 1860 à 1880 et dont le point culminant est très certainement la consécration de Félicité Angers. Lorsqu'elle entreprend une carrière littéraire à la fin des années 1870 sous le nom de Laure Conan, elle n'est pas aussi seule que l'histoire littéraire veut bien le laisser croire. Elle a, à ses côtés, des devancières et des contemporaines bien réelles, qui consacrent leurs loisirs à l'écriture, participent à des concours et publient dans les journaux.

${ }^{54}$ Jérôme Meizoz, Postures littéraires: mises en scène modernes de l'auteur, Genève, Slatkine, 2007, p. 25.

55 Daniel Mativat, Le métier d'écrivain au Québec (1840-1900): pionniers, nègres ou épiciers des lettres?, Montréal, Éditions Tryptique, 1996, p. 258. 